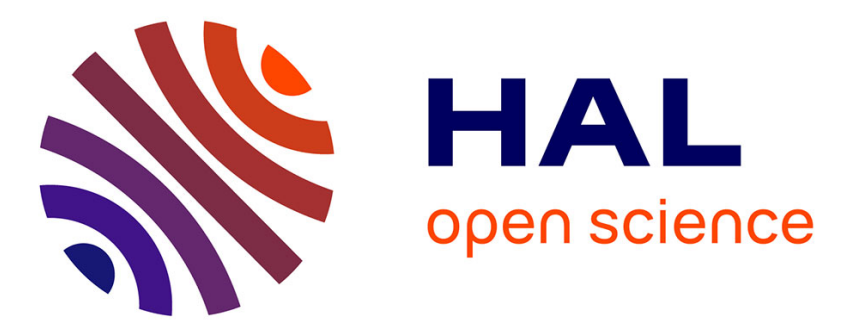

\title{
3D Modeling of Coils for Pulsed Field Magnetization of HTS Bulk Pellets in an Electrical Machine
}

Kévin Berger, Jakub Kapek, Alexandre Colle, Mariusz Stepien, Boguslaw Grzesik, Thierry Lubin, Jean Lévêque

\section{To cite this version:}

Kévin Berger, Jakub Kapek, Alexandre Colle, Mariusz Stepien, Boguslaw Grzesik, et al.. 3D Modeling of Coils for Pulsed Field Magnetization of HTS Bulk Pellets in an Electrical Machine. IEEE Transactions on Applied Superconductivity, 2018, 28 (4), pp.6801205. 10.1109/TASC.2018.2804906 . hal-01590665v2

\section{HAL Id: hal-01590665 \\ https://hal.science/hal-01590665v2}

Submitted on 8 Feb 2018

HAL is a multi-disciplinary open access archive for the deposit and dissemination of scientific research documents, whether they are published or not. The documents may come from teaching and research institutions in France or abroad, or from public or private research centers.
L'archive ouverte pluridisciplinaire HAL, est destinée au dépôt et à la diffusion de documents scientifiques de niveau recherche, publiés ou non, émanant des établissements d'enseignement et de recherche français ou étrangers, des laboratoires publics ou privés. 


\title{
3D Modeling of Coils for Pulsed Field Magnetization of HTS Bulk Pellets in an Electrical Machine
}

\author{
Kévin Berger, Jakub Kapek, Alexandre Colle, Mariusz Stepien, Boguslaw Grzesik, Thierry Lubin, and Jean Lévêque
}

\begin{abstract}
In this work, we propose to study and design a coil used to magnetize, by means of a Pulsed Field Magnetization (PFM) process, an inductor of a radial flux superconducting machine with one pair of poles. Each pole contains four similar HTS bulks of $30 \mathrm{~mm}$ diameter arranged in a square pattern. The cryostat already exists for this application and the temperature of the HTS bulks can vary from $4.2 \mathrm{~K}$ to their critical temperature, in transient state. For a given primary source of energy, here a capacitor bank of $10 \mathrm{~kJ}$ $(5 \mathrm{mF}, 2 \mathrm{kV})$ is available, the PFM process depends strongly on the value of the coil inductance used to generate pulsed field, because it defines the waveform of the current: peak value and time constant. Thus, 3D modeling of the coil is required in order to be sure that its inductance and the magnetic field produced will provide a full magnetization of HTS bulks. From the practical point of view, we would like to achieve an average magnetization of each pole around $3 \mathrm{~T}$.

In this paper, numerical modeling of coils with different number of turns coupled with circuit's equations is achieved. The maximum magnetic field obtained on the HTS bulks and estimated magnetization at the top center of each HTS bulk, are presented and discussed.
\end{abstract}

Index Terms-Bulk conductors, Inductance, Modeling, Pulse measurements, Superconducting rotating machines, Very high field and NMR magnets.

\section{INTRODUCTION}

$\mathbf{T}$ HE DEVELOPMENT of cryo-magnets based on REBaCuO bulk superconductors, that can generate up to $17 \mathrm{~T}$ [1], [2], gives the opportunity to see a technological breakthrough in electrical engineering developments. The key point to provide such development is the integration of the magnetization system including coils used to produce the magnetic field applied to the superconducting materials. Currently, the most convenient way to magnetize a High Temperature Superconducting (HTS) bulk is to use a pulsed magnetic field. This method is called PFM for Pulsed Field Magnetization [3], [4]. It can generate strong magnetic fields while using a relatively compact and simple coil. Thus, HTS bulks can be directly magnetized into the final application.

To the best of our knowledge, only a few teams have realized superconducting motors with HTS bulks acting as permanent magnets. As an example, the axial gap-type motor designed and realized in Japan can be mentioned here [5], [6]. This machine

The authors would like to acknowledge the "Région Lorraine" for its financial support. Jakub Kapek would like to thanks the Erasmus+ program for the funding of his internship in Nancy.

Kévin Berger, Jakub Kapek, Alexandre Colle, Thierry Lubin and Jean Lévêque are with the Group of Research in Electrical Engineering of Nancy, Faculty of Sciences and Technologies, University of Lorraine, 54506 Vandoeuvre-lèsNancy, France (e-mail: name.surname@univ-lorraine.fr). has been changed over the years to include now a double rotor and triple armatures [7], [8]. As another example, a radial type motor has also been realized in UK [9]. The stator consists of six air-core HTS armature windings and the rotor is made of 75 HTS bulks (15 columns, 5 pieces per column) mounted on surface and to be magnetized as a four-pole permanent magnet. [10]-[13]. All realized motors involving HTS bulks as permanent magnets have shown a trapped magnetic field by the HTS bulks lower than $1 \mathrm{~T}$. Therefore, the magnetization of the HTS bulks is still one of main scientific and technical challenge in such applications with the main goal to obtain a trapped magnetic flux density by the HTS bulks greater than $3 \mathrm{~T}$ [7], [14].

The analysis of electromagnetic, thermal and mechanical stresses on HTS bulks has not be studied in this paper. These difficult issues are the subject of recent studies started by means of numerical simulations [15], [16], and some experimental validations are underway.

Therefore, this paper deals with the design of the coils needed to magnetize HTS bulk pellets acting as the inductor of a superconducting electrical machine. The next section is devoted to present and describe the studied motor and to the foreseen specifications of the used coils. In Section III, the description of the 3D numerical model used to design the PFM coils is given, as well as the obtained results.

\section{MOTOR'S DESCRIPTION}

The activities of the GREEN laboratory are mainly dedicated to the possible applications of superconductivity in electrical engineering. In 2014, a new configuration of superconducting motor called "magnetic flux barrier motor" has been successfully designed and tested in the lab [17]-[20]. This motor, as shown in Fig. 1, has the particularity to include an inductor mounted inside a stationary cryostat and a rotating armature outside the cryostat.

The realization of a radial-type motor with magnetized HTS bulks acting as the inductor, is the next project of the GREEN, starting in October 2017. It was decided to keep the armature

Alexandre Colle is also with SAFRAN TECH., 78114 Magny-les-Hameaux, France.

Mariusz Stepien and Boguslaw Grzesik are with the Silesian University of Technology, Department of Power Electronics, Electrical Drives, 44-100 Gliwice, Poland. 


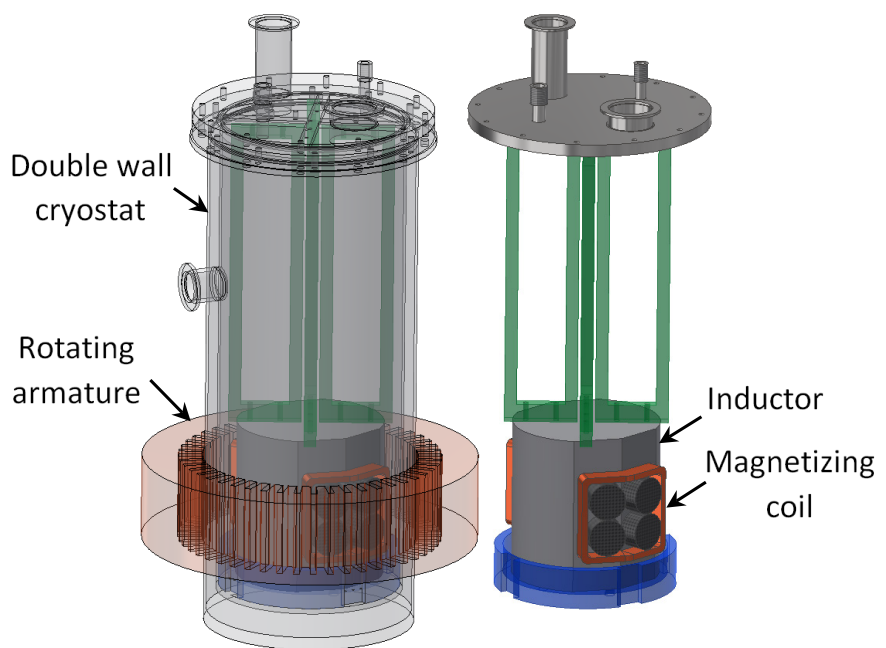

Fig. 1. Preliminary 3D drawings of the studied electrical machine.
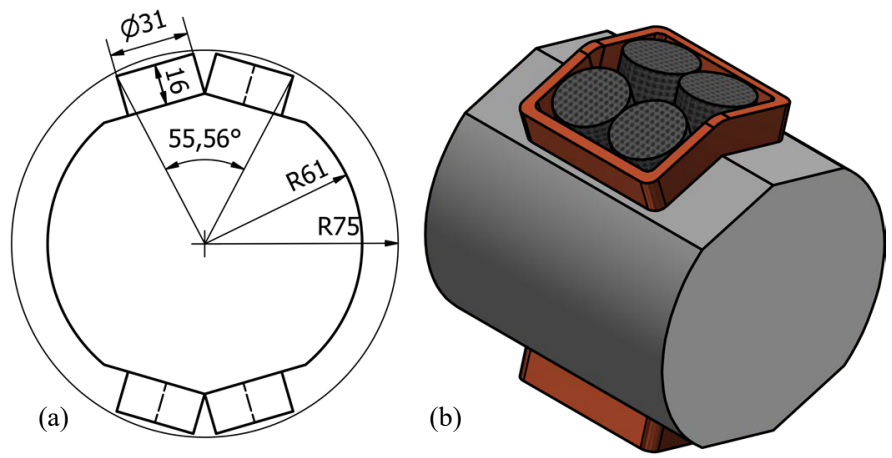

Fig. 2. Cross-sectional drawing of the inductor with given dimensions in $\mathrm{mm}$ (a) and a 3D isometric view with the magnetizing coils visible (b).

and cryostat of the previous motor, in order to avoid any extra cost.

\section{A. Description of the inductor}

The inductor will consist of one-pole pairs as shown in the previous realization. Each pole set contains four HTS bulks mounted on a square pattern and supposed to undergo the pulsed magnetization. We planned to use melt textured YBCO bulks from ATZ GmbH [21] or Can Superconductors [22]. These pellets are $16 \mathrm{~mm}$ high with a diameter of $31 \mathrm{~mm}$ and they can trap a maximal magnetic flux density of approximately $1.2 \mathrm{~T}$ at $77 \mathrm{~K}$ [22].

A cross-sectional drawing of the inductor including bulk pellets is shown in Fig. 2 (a), as well as a 3D isometric view in Fig. 2 (b). Some geometrical parameters of the motor are also given in Table I.

\section{B. Description of the impulse magnetizer}

In a previous experiment, it was possible to use a homemade setup with a bank of 24 capacitors with a total capacitance value of $80 \mathrm{mF}$ and voltage up to $300 \mathrm{~V}$ [23]. This setup, which also includes a thyristor, can withstand peak currents up to $15 \mathrm{kA}$. Recently, the lab acquired an impulse magnetizer from MagnetPhysik [24]. Here are the main technical characteristics: $10 \mathrm{~kJ}$ of stored energy, a capacitance of $5 \mathrm{mF}$, charging up to $2 \mathrm{kV}$, a peak surge current of $20 \mathrm{kA}$ and a non-repetitive peak current of $35 \mathrm{kA}$.
TABLE I

SOME GEOMETRICAL PARAMETERS OF THE MOTOR

\begin{tabular}{|c|c|c|}
\hline Symbol & Quantity & Value \\
\hline$R_{c y l}$ & $\begin{array}{l}\text { radius of the ferromagnetic cylinder } \\
\text { of the inductor }\end{array}$ & $61 \mathrm{~mm}$ \\
\hline$R_{\text {ind }}$ & $\begin{array}{l}\text { overall radius of the inductor } \\
\text { including the HTS bulks }\end{array}$ & $74 \mathrm{~mm}$ \\
\hline$e_{1}$ & $\begin{array}{l}\text { mechanical air gap between } \\
\text { the inductor and the cryostat }\end{array}$ & $1 \mathrm{~mm}$ \\
\hline$R_{\text {inn }}$ & inner radius of the cryostat & $75 \mathrm{~mm}$ \\
\hline$e_{2}$ & overall thickness of the cryostat & $12 \mathrm{~mm}$ \\
\hline$R_{\text {out }}$ & outer radius of the cryostat & $87 \mathrm{~mm}$ \\
\hline$e_{3}$ & $\begin{array}{l}\text { mechanical air gap between } \\
\text { the armature and the cryostat }\end{array}$ & $2 \mathrm{~mm}$ \\
\hline$R_{\text {arm }}$ & inner radius of the armature & $89 \mathrm{~mm}$ \\
\hline$e_{t}$ & $\begin{array}{l}\text { total air gap between the inductor } \\
\text { and the armature }\end{array}$ & $15 \mathrm{~mm}$ \\
\hline$L_{\text {arm }}$ & active length of the armature & $70 \mathrm{~mm}$ \\
\hline
\end{tabular}

These parameters are used in the next section to design the coil required to achieve the simultaneous magnetization of the four HTS bulks of one or both poles set, at the same time.

\section{Design of the Magnetizing CoIL}

\section{A. Description of the electrical system}

The equivalent electrical diagram of the impulse magnetizer system is given in Fig. 3. Once the capacitors are fully charged, the impulse current starts to flow in the magnetizing coil once the thyristor is switched on by the gate terminal. The time evolution of this current is governed by the RLC circuit parameters, for instance, the capacitance $C$, the initial charge voltage of the capacitors $U_{c 0}$, the resistance $R_{m c}$ and the inductance $L_{m c}$ of the magnetizing coil, the remaining resistance $R_{\lambda}$ and inductance $L_{\lambda}$ of the RLC circuit, e.g. cables.

All the mentioned parameters above are known except those of the magnetizing coil. The resistance $R_{m c}$ depends on the operating temperature of the coil and we assume that this temperature will never be higher than $100 \mathrm{~K}$ before starting the PFM process. At higher temperatures, the increase of the coil resistance leads to decrease drastically the peak current generated by the impulse magnetizer. The inductance $L_{m c}$ of the coil is shown as variable element in Fig. 3 because its value is nonlinear due to the presence of the ferromagnetic and superconducting materials.

One of the difficulties arising in the design of the magnetizing coil is that the current waveform and therefore, the maximum value of the magnetic field applied to the HTS bulks, depend on the non-linear inductance $L_{m c}$.

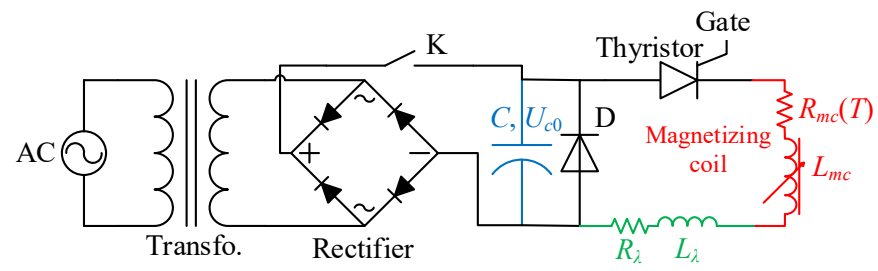

Fig. 3. Equivalent electrical diagram of the impulse magnetizer system used to magnetize HTS bulks. 


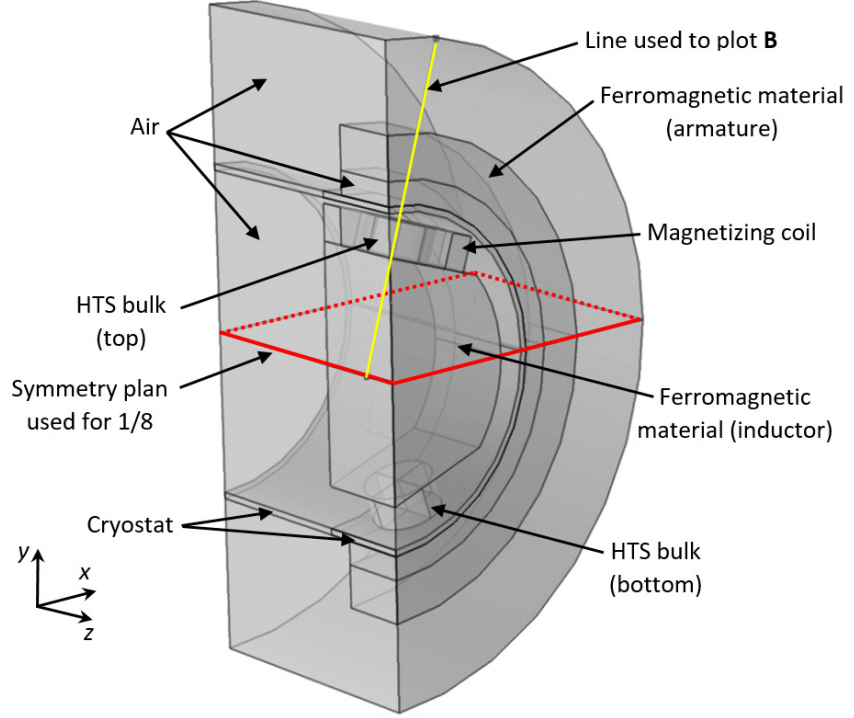

Fig. 4. 3D Geometry of the studied problem (1/4 of the geometry).

\section{B. 3D modeling of the magnetization of the inductor}

Since the magnetizing coil's geometry is three-dimensional and non-linear, the only way to design this coil is to use a numerical software. The widely used software, in particular by our research team in such studies, is the COMSOL ${ }^{\circledR}$ Multiphysics software [25]. It allows us to compute $3 \mathrm{D}$ electromagnetic problems using the magnetic field physics package and to couple the equations with the RLC circuit shown in Fig. 3.

In previous works, coils have successfully been realized for magnetizing one single HTS pellet by using copper foils of $0.2 \mathrm{~mm}$ or $0.5 \mathrm{~mm}$ thickness, and the same width as the HTS bulk height [23]. The coils to be modelled in the present study, will respect the same criteria. Kapton tape of $25 \mu \mathrm{m}$ thick is used between turns for insulation purpose. In order to optimize the magnetic flux produced by one pole, we have decided to design a single coil around the 4 HTS bulks of one pole instead of one coil around each bulk.

The inductor shown in Fig. 2 is made from a cobalt iron cylinder VACODUR ${ }^{\circledR} 50$ [26] including eight HTS bulks. Due to the symmetries, if we have to magnetize the four bulks of one pole, only a quarter $(1 / 4)$ of the geometry is needed to achieve the simulation. If both poles are magnetized at the same time, symmetries lead to study $1 / 8$ of the full geometry. Therefore, the studied problem with $1 / 4$ of the geometry is shown in Fig. 4. On the basis of these choices, parametric simulations have been carried out for a number of turns between 18 and 28 .

The current waveform for an initial capacitor voltage of $2 \mathrm{kV}$ is shown during the magnetization of one single pole in Fig. 5(a) and for both poles in series in Fig. 5(b). For 18 turns, the corresponding maximum current values $I_{\max }$ obtained during the magnetization of one pole and both poles, are respectively: $16.69 \mathrm{kA}$ and $11.97 \mathrm{kA}$. In both cases, the $I_{\max }$ value is still acceptable for the components of the electrical circuit. The decrease of the peak current is mainly due to the increase of the inductance of the coil when both coils are connected in series. Therefore, increasing the number of turns $N$ leads to a decrease of $I_{\max }$.
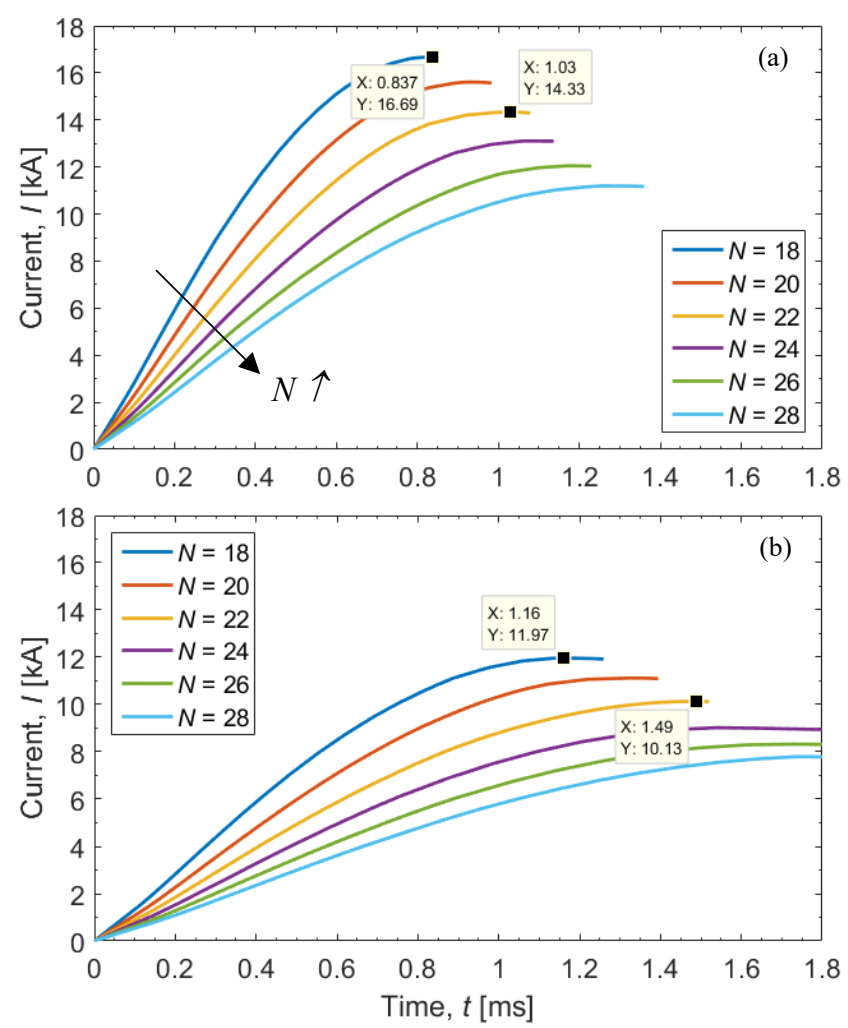

Fig. 5. Impulse current in the magnetizing coil for an initial capacitor voltage of $2 \mathrm{kV}$, during the magnetization of one single pole (a), and for both poles in series (b).
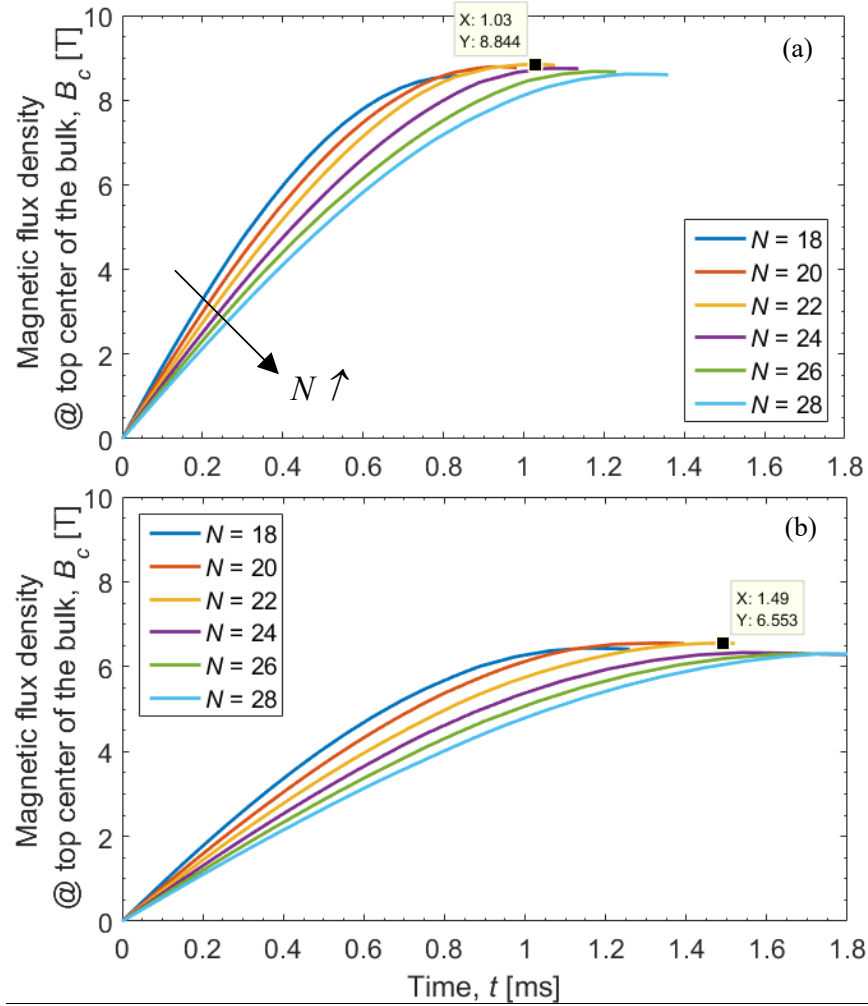

Fig. 6. Norm of the magnetic flux density $B_{c}$ at the top center of one HTS bulk, for an initial capacitor voltage of $2 \mathrm{kV}$, during the magnetization of one single pole (a), and for both poles in series (b)

Fig. 6 shows the norm of magnetic flux density $B_{c}$ at the top center of one HTS bulk, during the magnetization of one single pole in Fig. 6(a), and for both poles in series in Fig. 6 (b). For 
both configurations, there is an optimal value of magnetic flux density that we can apply to the HTS bulks. This maximum value of $B_{c}$ is obtained for 22 turns, while 20 turns give a very similar value. A maximum applied flux density of $8.844 \mathrm{~T}$ at the top center of the HTS bulk is obtained at $1.03 \mathrm{~ms}$, corresponding to a peak current of $14.33 \mathrm{kA}$, when one single pole is magnetized (see Fig. 6(a)). This maximum applied flux density is strongly decreased down to $6.553 \mathrm{~T}$ if both poles are magnetized simultaneously, as is shown in Fig. 6(b). Indeed, if both magnetizing coils are connected in series, the inductance increases which leads to a decrease of the peak current. For information purpose, the calculated inductance of one coil at 5 $\mathrm{ms}$ is $79.7 \mathrm{mH}$ compared to $167.3 \mathrm{mH}$ for both coils in series, almost twice as much, which means that there are not well coupled. Based on those results, and assuming a Bean's critical state model for the HTS bulks, a maximum trapped magnetic flux density around $4.4 \mathrm{~T}$, half of the maximum applied magnetic flux density, seems to be a manageable target.

Finally, the norm of the magnetic flux density and flux lines at the maximum current with $N=22$ are shown for the magnetization of one pole in Fig. 7 and the magnetization of both poles in Fig 8. The magnetic field distribution in the $x \mathrm{O} y$ plane at the middle of the HTS bulk are reported in Fig. 7(a) and Fig. 8(a), whereas Fig. 7(b) and Fig. 8(b) show the magnetic field distribution in the $x \mathrm{O} y$ plane at $x=0$. Fig. 7 and Fig. 8 give us the information about flux leakage and magnetic flux density amplitude in the whole inductor. The maximum magnetic flux density observed in Fig. 7 is around $18.3 \mathrm{~T}$ instead of $13.3 \mathrm{~T}$ in Fig. 8, while the flux lines crossing the air gap observed in Fig. 7 are much more numerous than obtained ones in the case shown by Fig. 8 .

\section{CONCLUSION}

The optimal design of a magnetizing coil for an inductor of a superconducting electrical machine with one pair of poles set of four HTS bulks each, has been achieved. The resulting coil consists of 22 turns of copper foil wound around the four pellets of each pole. The peak current of $14.33 \mathrm{kA}$ is obtained at $1.04 \mathrm{~ms}$ with produced magnetic flux density up to $8.844 \mathrm{~T}$ at the top center of the HTS bulk. From the practical point of view, to obtain the maximum magnetic flux density applied to the HTS bulk, the best process is to magnetize each pole one by one. Finally, we can expect a trapped magnetic flux density by the HTS bulks around $4.4 \mathrm{~T}$, which is a promising value compared to a state of the art in motors applications. The modelled magnetizing coil and the inductor in the present work, will be built during the next months and experimental results will give us confidence about the achievable trapped magnetic flux density for such application.

\section{ACKNOWLEDGMENT}

The authors would like to thank Rachid Dadi from VACUUMSCHMELZE GmbH for providing us with the VACODUR 50 material, and Salah Eddine Bentridi for the English proofreading of the final version of the paper.
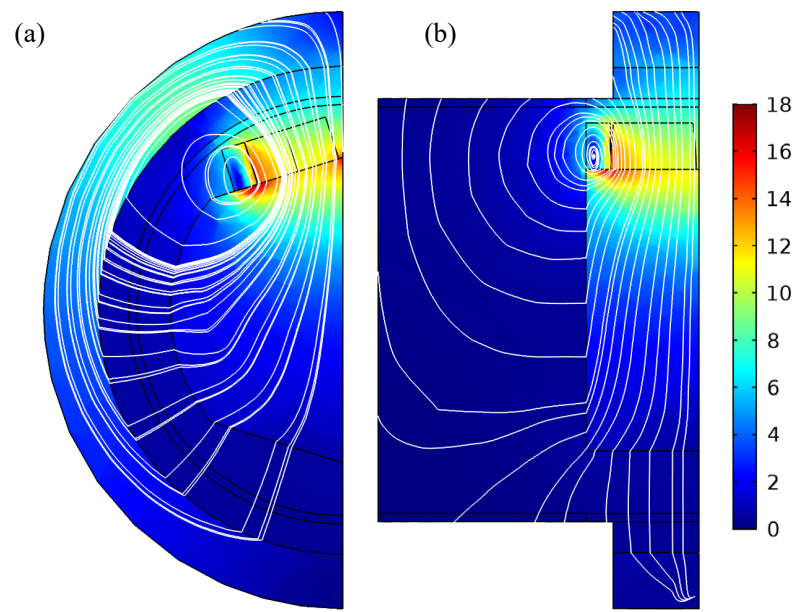

Fig. 7. Norm of the magnetic flux density in Tesla, and flux lines during the magnetization of one single pole with $N=22$ at $1.03 \mathrm{~ms}$, i.e. maximum current: in the $x \mathrm{O} y$ plane at the middle of the HTS bulk (a) and in the $y \mathrm{O} z$ plane $\mathrm{a} x=0(\mathrm{~b})$.
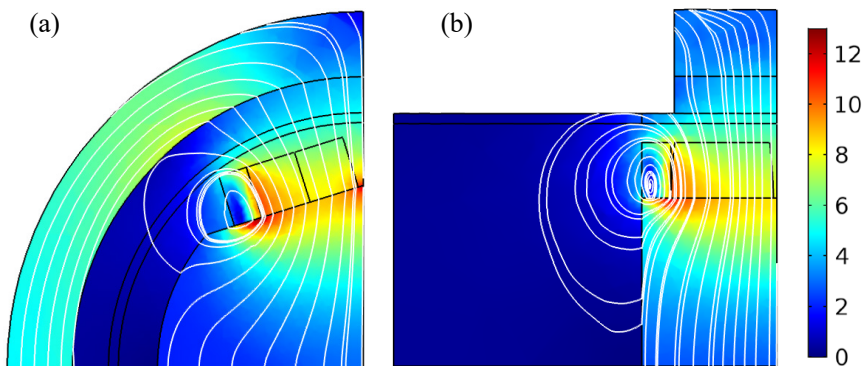

Fig. 8. Norm of the magnetic flux density in Tesla, and flux lines during the magnetization of both poles with $N=22$ at 1.49 ms, i.e. maximum current: in the $x \mathrm{O} y$ plane at the middle of the HTS bulk (a) and in the $y \mathrm{O} z$ plane $\mathrm{a} x=0$ (b).

\section{REFERENCES}

[1] J. H. Durrell et al., "A trapped field of $17.6 \mathrm{~T}$ in melt-processed, bulk GdBa-Cu-O reinforced with shrink-fit steel," Supercond. Sci. Technol., vol. 27, no. 8, p. 082001, 2014.

[2] M. Tomita and M. Murakami, "High-temperature superconductor bulk magnets that can trap magnetic fields of over 17 tesla at 29 K," Nature, vol. 421, no. 6922, pp. 517-520, Jan. 2003.

[3] U. Mizutani, T. Oka, Y. Itoh, Y. Yanagi, M. Yoshikawa, and H. Ikuta, "Pulsed-field magnetization applied to high-Tc superconductors," Appl. Supercond., vol. 6, no. 2, pp. 235-246, Feb. 1998.

[4] H. Fujishiro, T. Tateiwa, A. Fujiwara, T. Oka, and H. Hayashi, "Higher trapped field over 5T on HTSC bulk by modified pulse field magnetizing," Phys. C Supercond. Its Appl., vol. 445, pp. 334-338, Oct. 2006.

[5] H. Matsuzaki et al., "An axial gap-type HTS bulk synchronous motor excited by pulsed-field magnetization with vortex-type armature copper windings," IEEE Trans. Appl. Supercond., vol. 15, no. 2, pp. 2222-2225, Jun. 2005.

[6] M. Miki et al., "Development of a synchronous motor with $\mathrm{Gd}-\mathrm{Ba}-\mathrm{Cu}-$ O bulk superconductors as pole-field magnets for propulsion system," $\mathrm{Su}$ percond. Sci. Technol., vol. 19, no. 7, p. S494, 2006.

[7] Y. Zhang, D. Zhou, T. Ida, M. Miki, and M. Izumi, "Melt-growth bulk superconductors and application to an axial-gap-type rotating machine," Supercond. Sci. Technol., vol. 29, no. 4, p. 044005, 2016.

[8] H. Matsuzaki et al., "HTS Bulk Pole-Field Magnets Motor With a Multiple Rotor Cooled by Liquid Nitrogen," IEEE Trans. Appl. Supercond., vol. 17, no. 2, pp. 1553-1556, Jun. 2007.

[9] Q. Jiang, M. Majoros, Z. Hong, A. M. Campbell, and T. A. Coombs, "Design and AC loss analysis of a superconducting synchronous motor," $\mathrm{Su}$ percond. Sci. Technol., vol. 19, no. 11, p. 1164, 2006. 
[10] Z. Hong, W. Yuan, M. Ainslie, Y. Yan, R. Pei, and T. A. Coombs, “AC Losses of Superconducting Racetrack Coil in Various Magnetic Conditions," IEEE Trans. Appl. Supercond., vol. 21, no. 3, pp. 2466-2469, Jun. 2011.

[11] W. Xian, Y. Yan, W. Yuan, R. Pei, and T. A. Coombs, "Pulsed Field Magnetization of a High Temperature Superconducting Motor," IEEE Trans. Appl. Supercond., vol. 21, no. 3, pp. 1171-1174, Jun. 2011.

[12] Z. Huang, M. Zhang, W. Wang, and T. A. Coombs, "Trial Test of a BulkType Fully HTS Synchronous Motor,” IEEE Trans. Appl. Supercond., vol. 24, no. 3, pp. 1-5, Jun. 2014.

[13] Z. Huang, H. S. Ruiz, W. Wang, Z. Jin, and T. A. Coombs, "HTS Motor Performance Evaluation by Different Pulsed Field Magnetization Strategies," IEEE Trans. Appl. Supercond., vol. 27, no. 4, pp. 1-5, Jun. 2017.

[14] K. Berger et al., "High Magnetic Field Generated by Bulk MgB2 Prepared by Spark Plasma Sintering," IEEE Trans. Appl. Supercond., vol. 26, no. 3, pp. 1-5, Apr. 2016.

[15] F. Trillaud, K. Berger, B. Douine, and J. Leveque, "Distribution of current density, temperature and mechanical deformation in YBCO bulks under Field-Cooling magnetization," IEEE Trans. Appl. Supercond., vol. PP, no. 99, pp. 1-1, 2018.

[16] K. Takahashi, H. Fujishiro, T. Naito, Y. Yanagi, Y. Ito, and T. Nakamura, "Numerical simulation of electromagnetic and thermal stress in RE$\mathrm{BaCuO}$ superconducting ring and disk bulks reinforced by stainless steel ring with various widths during field-cooled magnetization," IEEE Trans. Appl. Supercond., vol. PP, no. 99, pp. 1-1, 2018.

[17] R. Alhasan et al., "Study of a superconducting motor with high specific torque," in MEA 2015 More Electric Aircraft, Toulouse, France, 2015, p. 91.

[18] R. Alhasan, T. Lubin, Z. M. Adilov, and J. Lévêque, "A New Kind of Superconducting Machine,” IEEE Trans. Appl. Supercond., vol. 26, no. 3, pp. 1-4, Apr. 2016.
[19] R. Alhasan, T. Lubin, B. Douine, Z. M. Adilov, and J. Lévêque, "Test of an Original Superconducting Synchronous Machine Based on Magnetic Shielding," IEEE Trans. Appl. Supercond., vol. 26, no. 4, pp. 1-5, Jun. 2016.

[20] M. Kelouaz et al., "3D Magnetic field modeling of a new superconducting synchronous machine using reluctance network method," Phys. C Supercond. Its Appl., vol. 548, pp. 5-13, May 2018.

[21] F. Werfel, "Adelwitz Technologiezentrum GmbH," ATZ, 11-May-2016. [Online]. Available: http://www.atz-gmbh.com/. [Accessed: 11-May2016].

[22] "CSYL-35 YBCO Levitation Disk - CAN SUPERCONDUCTORS eshop." [Online]. Available: http://shop.can-superconductors.com/index.php?id_product $=15 \&$ controller=product. [Accessed: 08-Sep-2017].

[23] B. Gony, K. Berger, B. Douine, M. R. Koblischka, and J. Leveque, "Improvement of the Magnetization of a Superconducting Bulk using an Iron Core," IEEE Trans. Appl. Supercond., vol. 25, no. 3, pp. 1-4, Jun. 2015.

[24] "MAGNET-PHYSIK Dr. Steingroever GmbH: Impulse Magnetizer." [Online]. Available: http://www.magnet-physik.de/impulsmagnetisierer.html?L=1. [Accessed: 09-Sep-2017].

[25] K. Berger et al., "Benchmark on the 3D Numerical Modeling of a Superconducting Bulk," in 21st International Conference on the Computation of Electromagnetic Fields (Compumag 2017), Daejeon, South Korea, 2017, p. (ID 110).

[26] "VACODUR - Magnetic Properties - VACUUMSCHMELZE GmbH \& Co. KG." [Online]. Available: http://www.vacuumschmelze.com/en/products/materials-parts/soft-magnetic/cobalt-iron/vacodur/vacodur-magnetic-properties.html. [Accessed: 16-Sep-2017]. 